\title{
Analyse des contraintes résiduelles et de la texture dans les matériaux : applications industrielles
}

\author{
P.J. Webster
}

Institut d'Ingénieurerie des Matériaux, École en Aéronautique, Génie Civil et Mécanique, Université de Salford, Manchester, U.K.

\section{I- INTRODUCTION : LES CONTRAINTES RESIDUELLES}

\section{II- DETERMINATION DES CONTRAINTES RESIDUELLES}

II.1- Déformations et contraintes

II.2- Méthodes expérimentales

II.3- Les techniques de diffraction

II.3.1 - La théorie des méthodes de diffraction

II.3.2- Le balayage traditionnel des déformations aux rayons $\mathrm{X}$

II.3.3- Le balayage des déformations par neutrons

II.3.4- Le balayage des déformations par rayons $\mathrm{X}$ obtenus par synchrotrons

III- ETUDE DE CAS- RAYONS X OBTENUS PAR SYNCHROTRONS, NEUTRONS

III.1 - Les rails de chemin de fer

III.2- Soudure d'acier ferritique

III.3- Alliages d'aluminium soumis au grenaillage

III.4- Trou agrandi à froid

III.5- Soudure en «double V»- Micro-déformations

\section{IV- CONCLUSION}

Remerciements

\section{V- REFERENCES}

\section{Résumé}

Les contraintes résiduelles s'introduisent lors de l'ingénierie des composants au cours de leur fabrication et également au cours de leur utilisation. Elles sont importantes parce qu'elles peuvent avoir des magnitudes proches de la limite apparente d'élasticité et peuvent par conséquent affecter considérablement la performance et la durée de vie des composants. Les ingénieurs concepteurs doivent être en mesure de calculer et de mesurer les champs de contraintes résiduelles afin de s'assurer de l'intégrité des composants. Cet article décrit les techniques expérimentales utilisées pour déterminer des champs de contraintes résiduelles en faisant référence plus précisément à la diffraction des neutrons et à la diffraction des rayons $\mathrm{X}$ obtenus par synchrotrons. On y discute de la théorie et de l'applicabilité de ces techniques. Cet article comprend un certain nombre de cas étudiés à titre d'exemple. Il renvoie aussi à l'évolution des étalons internationaux de mesure de contraintes résiduelles et aux développements à venir.

\section{Introduction : Les Contraintes Résiduelles}

Des contraintes résiduelles sont présentes dans la plupart des composants de l'ingénierie. Ce sont les contraintes qui demeurent même après que toutes les interactions extérieures aient été 
supprimées. Ces contraintes sont généralement présentes dans les composants qui ont subi un fléchissement en certains points à cause d'une application non uniforme de contraintes mécaniques ou thermiques. Lorsque les contraintes appliquées sont supprimées, le composant tente de se détendre de façon élastique, mais il reste contraint à cause des régions du composant qui ont subi une déformation plastique. Tant et si bien que, bien que la contrainte soit de nouveau uniformément équilibrée, certaines régions du composant se retrouvent dans un état de contrainte résiduelle de traction ou de compression.

Les contraintes résiduelles peuvent avoir des ampleurs proches de la limite apparente d'élasticité et affecter de manière significative les performances et la durée de vie des composants fatigués parce que la contrainte totale subie par un composant est égale à la somme des contraintes appliquées et des contraintes résiduelles. Si les contraintes résiduelles et les contraintes appliquées sont du même signe, leur somme a une grandeur plus importante que celle de l'une ou l'autre des contraintes résiduelles ou appliquées - ceci peut être une cause suffisante de rupture apparente d'élasticité. A l'inverse, si leurs signes sont opposés, leur somme sera moins importante, et les effets peuvent même être bénéfiques. Que les conséquences soient positives ou négatives, elles sont considérables et, par conséquent, elles sont d'une importance primordiale pour l'ingénierie. En général, les champs de contraintes résiduelles correspondront à des compressions dans les régions plastiquement déformées par une traction et à des tractions dans les régions plastiquement déformées par une compression. Dans certains cas, des champs de contraintes résiduelles sont délibérément produits en tant que partie intégrante du processus de fabrication afin de prévenir l'apparition et la propagation de fissures dues à la fatigue. Un exemple illustrant ce cas de figure : le grenaillage où on bombarde une surface avec des petites billes pour créer une déformation plastique de traction en surface. La déformation qui en résulte crée une couche en compression proche de la surface qui prévient la propagation de fissures dues à la fatigue et aux défauts de surface. Le grenaillage est largement utilisé dans l'industrie aérospatiale pour prolonger la durée de vie des composants des moteurs d'avion et des fuselages. Des contraintes résiduelles dues à des variations thermiques peuvent résulter des écarts de refroidissement qui adviennent dans des processus tels que la soudure. D'habitude, les contraintes résultantes de soudure sont nuisibles car elles peuvent générer des distorsions ou des pannes prématurées dues à la fatigue. Elles doivent être réduites par des traitements thermiques ou mécaniques post-soudure qui peuvent, eux-même, augmenter les coûts de fabrication de façon significative. En outre, les parties composantes qui sont soumises à des cycles de fatigue thermo-mécaniques, telles que les moteurs, ou qui sont soumises à des contraintes mécaniques de contact répété, telles que les rails de chemin de fer, verront leurs champs de contraintes résiduelles modifiés au cours de leur utilisation.

Indépendamment de la façon dont les contraintes résiduelles sont générées, il est absolument essentiel pour les ingénieurs de savoir ce qu'elles sont afin qu'ils puissent incorporer l'information dans leurs projets et estimer de façon fiable les performances et la durée de vie des composants. D'autant plus lorsqu'il s'agit de composants essentiels à la sécurité. Typiquement c'est le cas des disques et des pales de moteurs d'avions qui sont soumis à de sévères cycles de fatigue thermo-mécanique lorsqu'ils sont en marche, aussi sont-ils soumis au grenaillage afin de prolonger leur durée de vie.

\section{Détermination des contraintes résiduelles}

\subsection{Déformations et Contraintes}

En tout point donné, les contraintes résiduelles sont déterminées par un calcul à partir des déformations résiduelles en ce point et en utilisant les valeurs appropriées du module de 
Young et du coefficient de Poisson. En général, pour définir la déformation en un point, des mesures exhaustives sont nécessaires dans six directions indépendantes au moins. En pratique, si les directions principales sont connues, trois suffisent. Lorsque les directions principales coïncident avec les directions des coordonnées orthogonales, les contraintes principales $\sigma$ sont reliées aux déformations $\varepsilon$ par l'équation:

$$
\sigma_{\mathrm{x}}=\mathrm{E}\left[(1-v) \varepsilon_{\mathrm{x}}+v\left(\varepsilon_{\mathrm{y}}+\varepsilon_{\mathrm{z}}\right)\right] /[(1+v)(1-2 v)]
$$

impliquant des équations équivalentes pour $\sigma_{y}$ et $\sigma_{z}$. E correspond au module de Young et $v$ correspond au coefficient de Poisson.

\subsection{Méthodes Expérimentales}

Les méthodes de mesure des déformations résiduelles se divisent grossièrement en trois catégories: les méthodes mécanique, physique et par diffraction. Avec les méthodes mécaniques, de la matière est ôtée au composant par découpage ou par forage. Le relâchement qui en résulte est mesuré, généralement par des jauges de déformation de surface et la tension résiduelle de départ est alors calculée. Ces méthodes sont complètement ou partiellement destructrices et comprennent la méthode de forage de Sachs, la perforation (progressive et profonde), la trépanation, le sectionnement et l'entaillage.

Les méthodes physiques sont généralement non-destructrices et comprennent des méthodes acoustiques, magnétiques et optiques.

Les méthodes par diffraction comprennent les méthodes traditionnelles de retrodiffusion des rayons $\mathrm{X}$, les neutrons et les rayons $\mathrm{X}$ obtenus par synchrotrons.

Toutes ces méthodes ont leurs avantages et leurs limites. Les calculs nécessaires pour la méthode mécanique de suppression de matière peuvent être difficiles ou douteux lorsque l'échantillon étudié est de forme complexe. Cependant les méthodes mécaniques sont très largement employées et tout à fait appropriées à de nombreuses applications. Les méthodes ultrasonores et électromagnétiques sont utiles quand il s'agit d'identifier rapidement des problèmes d'homogénéité, des défauts et des points de tension élevée ; mais elles sont moins efficaces quand il s'agit de dresser une carte quantitative des déformations directionnelles. Les techniques par diffraction évoluent rapidement au fur et à mesure que des sources nouvelles et plus intenses permettent une utilisation plus efficace et générale. Les méthodes traditionnelles attachées aux rayons $X$ sont utiles dans le cas de mesure de surface. Les neutrons sont utilisés depuis les années 1980 pour prendre des mesures internes et nondestructrices grâce à leur pouvoir de pénétration [1-9]. Des méthodes liées à l'utilisation de rayons $\mathrm{X}$ obtenus par synchrotrons dont l'intensité est plus importante sont en train de se développer afin de fournir des données complémentaires sur les déformations résiduelles internes de façon non-destructrice - et ce, en particulier dans le cas de matériaux contenant des éléments légers, d'intérêt général pour l'aérospatiale [10-16].

\subsection{Les Techniques de Diffraction}

\subsubsection{LA THÉORIE DES MÉTHODES DE DIFFRACTION}

Les méthodes de diffraction sont applicables à des matériaux polycristallins. Elles mesurent les changements de dimensions du réseau qui résultent des contraintes appliquées ou résiduelles en fonction de leur emplacement et de leur direction. Le réseau cristallin est utilisé comme une jauge des déformations internes à trois dimensions, sondée par un faisceau de rayons $\mathrm{X}$ ou de neutrons d'une longueur d'onde comparable aux espacements du réseau. 
Un changement $\delta \mathbf{d}$ dans la distance d'un ensemble donné de plans du réseau, qui, au départ, ne présente pas la moindre tension et présente un espacement $\mathbf{d}$, produira un changement correspondant $\delta \theta$ dans la position de la réflexion de Bragg, qui se présentait à l'origine sous un angle $\theta$, ce changement est relié aux déformations $\varepsilon$ du réseau par l'équation suivante :

$$
\varepsilon=\delta \mathrm{d} / \mathbf{d}=-\cot \theta \cdot \delta \theta
$$

Le léger changement, $\delta 2 \theta$, dans l'angle du faisceau diffracté, dispersé à partir d'un petit "volume témoin" de l'échantillon, est mesuré en fonction de la position et de la direction dans l'échantillon. Le changement mesuré est converti en déformation en employant l'équation 2 et les déformations dans les diverses orientations sont combinées par l'emploi de l'équation 1 ou son équivalent pour déterminer les contraintes.

La sensibilité aux déformations de la méthode dépend de l'angle et est déterminée par l'équation suivante:

$$
-\delta \theta / \varepsilon=\tan \theta
$$

Typiquement, les déformations sont mesurées dans une fourchette allant de $10^{-5}$ à $10^{-2}$ et donc les angles $\delta \theta$ mesurés sont très petits. Les neutrons sont généralement détectés autour de $90^{\circ}$, angle pour lequel la sensibilité aux déformations est de 1,0. Aux angles les plus grands, utilisés typiquement pour des mesures traditionnelles au rayons $X$ retrodiffusés, il y a la plupart du temps une augmentation de la sensibilité d'environ $\times 10$. Mais pour des dispersions selon les angles les plus faibles, typiques de mesures qui utilisent des rayons $\mathrm{X}$ durs obtenus par synchrotrons, la sensibilité décroît à environ un dixième de la valeur qu'elle a à $90^{\circ}$.

\subsubsection{LE BALAYAGE TRADITIONNEL DES DEFOPRMATIONS AUX RAYONS X}

La plupart des balayages des déformations par rayons $\mathrm{X}$ se fait en utilisant la radiation caractéristique $\mathrm{K} \alpha$ de générateurs portables ou fixes dans des laboratoires. Aux angles proches de la retrodiffusion la sensibilité à la déformation est élevée. Par conséquent, des mesures prises à un centième de degré près sont généralement suffisantes. Le doublet Ka peut être résolu comme il peut aussi ne pas l'être, ceci peut compliquer sinon limiter la fiabilité de la détermination de la position du sommet du pic de diffraction.

La contrainte majeure à l'étude par balayage des déformations grâce aux rayons X est l'atténuation du faisceau par l'échantillon. L'atténuation des rayons $X$ varie en fonction de la longueur d'onde et du nombre atomique. Pour des longueurs d'ondes d'environ $1.5 \AA$ dans la plupart des matériaux de l'ingénierie elles sont relativement élevées, typiquement de l'ordre de $0.01 \mathrm{~mm}$. Par conséquent cette technique se limite généralement à la dispersion en retrodiffusion à partir d'épaisseurs de surface de l'ordre de 10 microns soit l'ordre de l'épaisseur représentée par la figure 1. Les déformations dans le plan sont obtenues à partir d'une série de mesures faites en basculant l'échantillon et à partir de calculs qui emploient la méthode $\sin ^{2} \psi$. 


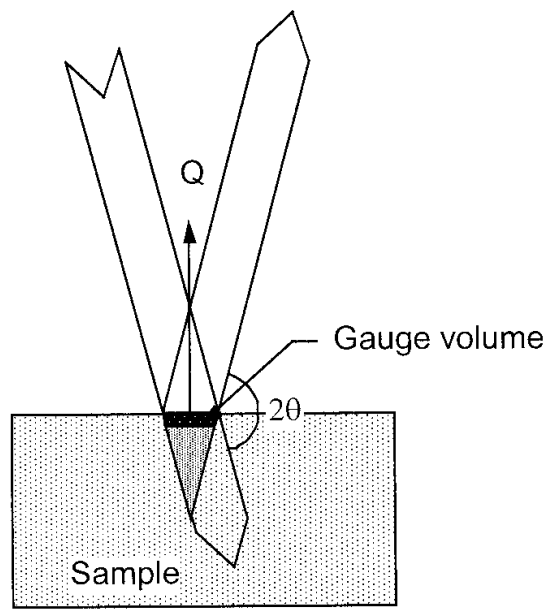

Figure 1 : La configuration du balayage traditionnel des déformations par rayons $X$ à faible retour

La méthode est bien établie et largement utilisée dans l'industrie pour les mesures de surface en laboratoire et sur le terrain. Si un profil détaillé en fonction de la profondeur du champ des contraintes résiduelles proches de la surface est nécessaire, il faut généralement avoir recours à des méthodes d'érosion chimique afin d'exposer aux rayons $\mathrm{X}$ les couches sous la surface. Comme pour les méthodes mécaniques, des corrections doivent alors être faites pour tenir compte des effets de relaxation dus à la suppression d'épaisseurs.

\subsubsection{LE BALAYAGE DES DEFORMATIONS PAR NEUTRONS}

Par rapport aux rayons X de $1.5 \AA$, les neutrons, qui ont des longueurs d'ondes semblables, sont bien plus pénétrants et ont des longueurs d'atténuation typiquement de l'ordre de $10 \mathrm{~mm}$ pour les matériaux d'ingénierie les plus communs. Il est donc possible, avec les neutrons, de prendre des mesures en transmission au travers de plusieurs millimètres ou même centimètres de matériau. Des mesures sont régulièrement prises au travers d'échantillons d'acier de $25 \mathrm{~mm}$ d'épaisseur. Cette méthode est utilisée en recherche depuis 1980 environ mais elle commence à être plus largement adoptée par les industries de pointe et un standard international est en préparation. C'est généralement une technique efficace pour un balayage à définition moyenne des déformations internes en plusieurs points. C'est aussi une technique non destructrice. L'ébauche d'une configuration typique de diffusion des neutrons avec une configuration proche de $90^{\circ}$ est représente en figure 2 .

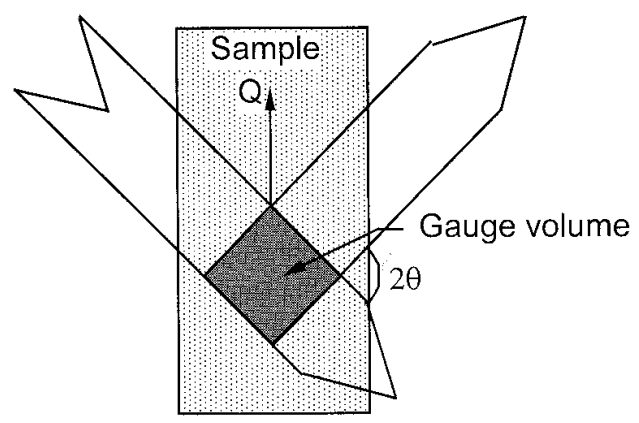

Figure 2 : Configuration d'une étude par balayage des déformations par diffraction des neutrons sous un angle proche de $90^{\circ}$ 
Dans une étude par balayage des déformations par neutrons à partir d'une source intense, comme un réacteur nucléaire par exemple, un faisceau de neutrons est collimaté puis monochromaté par un cristal, enfin masqué pour donner un faisceau étroit dont on détermine la section efficace. Une fente est placée devant le détecteur afin de définir la direction du faisceau et la troisième dimension de la jauge. La réflexion de Bragg et la longueur d'onde sont généralement choisies de façon à ce que le détecteur puisse être positionné à un angle proche de $90^{\circ}$. Cela donne une résolution spatiale optimale pour la plupart des séries de mesures orthogonales. Typiquement, les déplacements des pics de diffraction à cet angle sont de l'ordre de quelques dixièmes de degré avec une précision de quelques millièmes de degré. Pour des mesures internes de routine dans des composants dont les variations de déformations sont modérées, un volume de jauge typique sera un cube de $2 \mathrm{~mm}$ de côté. Pour les mesures de champs de tension dont les gradients sont forts, et les mesures proches de la surface, des jauges plus petites, d'environ $0,5 \mathrm{~mm}$ de côté sont utilisées. Si les gradients sont faibles dans une direction donnée, les dimensions de la jauge peuvent être augmentées pour réduire le nombre des calculs à faire et améliorer les statistiques. La fréquence des mesures peut varier de plus d'une par minute à une par heure et par pic de diffraction. Des détecteurs multiples sont utilisés pour améliorer la fiabilité des calculs mais ils peuvent aussi introduire des aberrations de surface[9] si les techniques appropriées ne sont pas employées.

Les neutrons ont été utilisés dans les études d'un large éventail de matériaux et de composants d'ingénierie: des rails de chemin de fer, des soudures, des chaînes en anneaux, des tiges contraintes, des surfaces traitées et soumises au grenaillage [1-9]. Elle fut la première des techniques utilisées pour observer les contraintes résiduelles proches de la surface dans les alliages spaciaux fortement grenaillés. Le projet VAMAS intitulé TWA20 ou 'Mesures des contraintes résiduelles' a été lancé en 1996 afin d'établir les bases scientifiques du balayage des déformations par neutron et pour esquisser un standard pour son utilisation et son application industrielle. Le projet est censé atteindre ce premier objectif en 2001, après quoi il est prévu que l'utilisation industrielle du balayage des déformations par neutrons augmentera sensiblement.

\subsubsection{LE BALAYAGE DES DÉFORMATIONS PAR RAYONS X OBTENUS PAR SYNCHROTRONS}

Au début des années 1990 le synchrotron de Daresbury SRS a été utilisé à une longueur d'onde de rayons $\mathrm{X}$ de $0.5 \AA$ pour étudier des variations en profondeur du champ des contraintes résiduelles dans une plaque d'aluminium de $1.6 \mathrm{~mm}$ d'épaisseur ayant été soumise au grenaillage sur une de ses faces et ayant été légèrement façonnée de l'autre [1014]. La fréquence des mesures en chacun des points était d'une toutes les minutes. Les résultats avaient démontré le potentiel de la méthode et le besoin de développements significatifs, pour la rendre plus exploitable. Depuis lors, des avancées certaines ont été faites notamment à l'ESRF de Grenoble.

La figure 3 illustre une coupe transversale d'une jauge à angle faible telle qu'on pourrait en utiliser dans le balayage des déformations par synchrotrons. 


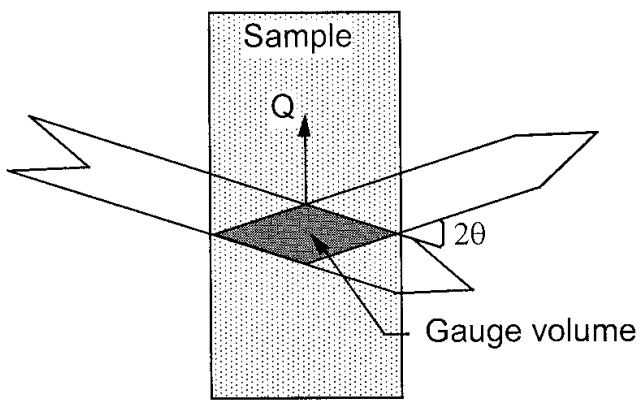

Figure 3 : Configuration du balayage des déformations par synchrotrons pour un petit angle de transmission.

L'utilisation de longueurs d'ondes plus courtes encore et les sources de synchrotrons modernes permettent de faire des mesures par rayons $\mathrm{X}$ avec une haute définition spatiale, au travers d'échantillons de plus en plus denses et épais. On prévoit que les techniques basées sur les rayons $\mathrm{X}$ obtenus par synchrotrons, et sur les neutrons, vont devenir des techniques capables de dessiner des cartes de champs de déformations résiduelles internes pour un large panel de composants et de matériaux d'ingénierie.

\section{Etude de cas - Rayons $\mathrm{X}$ produits par synchrotrons, neutrons}

\subsection{Les rails de chemin de fer}

Les contraintes résiduelles qui sont introduites dans les rails de chemin de fer au cours du processus de fabrication, puis qui sont modifiées au cours de leur mise en service et de leur emploi, ont une influence importante sur la fréquence et les mécanismes de défaillance des rails. La pression répétée de charges dues aux roues du train peut générer une fatigue due au contact roulant qui peut aboutir à des fissures, un effrittement, et même à la fracture du rail. Les techniques mécaniques de relaxation, qui sont souvent utilisées dans le cadre de mesures de déformations dans les rails, sont destructrices et donc restreintes à un petit nombre de mesures de surface. La diffraction par neutrons est capable de fournir des données de haute qualité, non-destructrices, et tri-dimensionnelles sur les déformations internes qui sont appropriées à la validation de modèles numériques.

Des mesures par neutrons ont été faites sur un échantillon d'un nouveau rail dont la tête a été durcie et aplatie au rouleau dénommé $370 \mathrm{BHN}$, pour le programme d'essais Européen ORE $\mathrm{D}$ 173. De façon à réduire l'atténuation à des niveaux raisonnables, des sections de $10 \mathrm{~mm}$ de long et de large ont été coupées dans le rail comme le montre la figure 4. Même si ces sections présentaient un relâchement significatif dans la direction transversale, elles retenaient tout de même des déformations non négligeables dans les autres directions. 


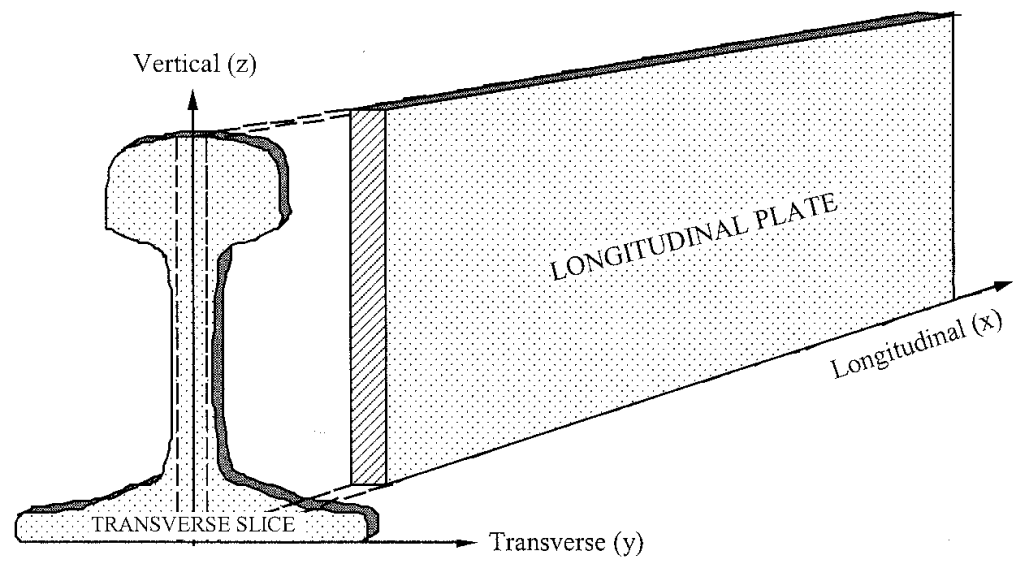

Figure 4: Echantillons de la section transverse et de la plaque longitudinale prélevés dans un rail[7]

Des mesures ont été faites le long de la ligne centrale de la plaque longitudinale, dans les directions verticale, transversale, et longitudinale en utilisant le diffractomètre D20 à l'ILL de Grenoble [7]. Le volume de la jauge était de $2 \mathrm{~mm}$ de côté. Les contraintes résiduelles longitudinales dérivées des données des déformations sont données en figure 5 avec les données obtenues par la méthode mécanique de sectionnement. Les valeurs de surface coïncident mais il y a des différences dans les valeurs internes. La plus évidente de ces différences dans les résultats obtenus est la quantité de points de mesure. Le balayage à neutrons révèle un détail fin que ne montre pas la méthode mécanique et de plus il est possible de répéter les mesures avec le balayage à neutrons.

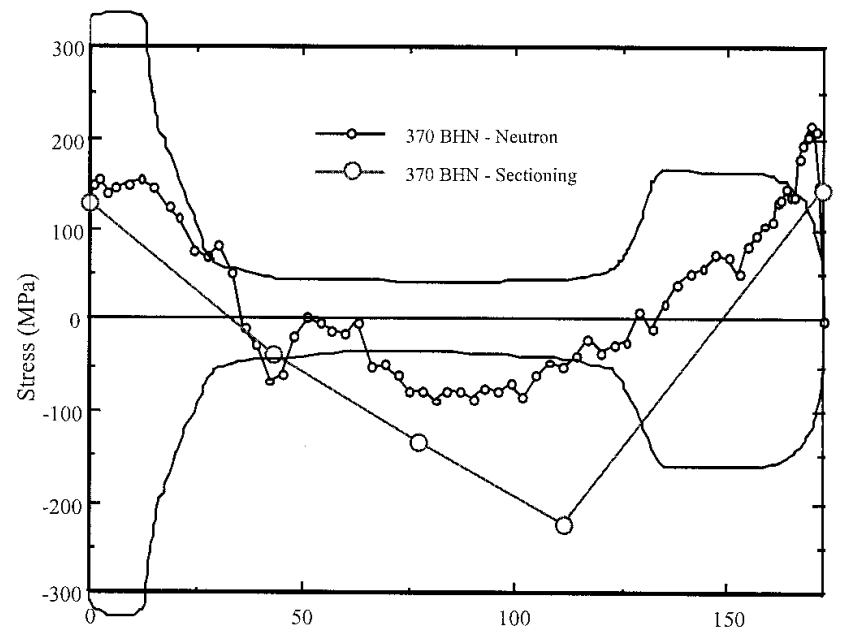

Figure 5: Les contraintes longitudinales dans les nouveaux rails 370 BHN mesurées par les méthodes de sectionnement et par le balayage à neutrons [7] 


\subsection{Soudure d'Acier Ferritique}

L'acier ferritique, étant solide, abondant et relativement peu coûteux, est probablement le métal le plus utilisé dans l'industrie de la construction. Dans la construction, il doit souvent être raccordé par des attaches mécaniques ou bien par soudure. La soudure est souvent l'option la moins chère pour effectuer des joints permanents mais elle peut aussi générer un champ de contraintes résiduelles intense et à gradient élevé, qui peut à son tour générer des distorsions et réduire la durée de vie de la structure.

Des mesures par balayage à neutrons ont été faites sur une soudure «multi-pass » faite ellemême dans une rainure en forme de $\mathrm{U}$ de $8 \mathrm{~mm}$ d'épaisseur ; la rainure suivant le long de la ligne centrale d'une plaque d'acier ferritique d'un mètre de long, de $15 \mathrm{~mm}$ de large et de 12,5 mm d'épaisseur. La soudure était fournie par TWI, à Abington au R.U., en tant qu'échantillon test pour le projet international VAMAS TWA20 ; projet mis en place pour définir le premier standard international de détermination des contraintes résiduelles par la technique de diffraction par neutrons. Les mesures ont été faites selon trois directions orthogonales : le long de la direction de la soudure $(X)$, sur la perpendiculaire dans le plan $(\mathrm{Y})$ et selon la direction normale au plan $(\mathrm{Z})$. Pour ces mesures on a utilisé les équipements du réacteur nucléaire du NRC de Chalk River au Canada. Toutes les mesures ont été prises sur la coupe transversale $\mathrm{YZ}$ au milieu de l'échantillon. Chaque mesure a pris, respectivement, entre $30 \mathrm{sec}$. et $5 \mathrm{~min}$., selon les dimensions du volume de la jauge utilisée. Les résultats des balayages linéaires sont schématisés respectivement par les figures 6 et 7 $[15,16]$. Le diagramme des déformations résiduelles, dans la figure 6 , est quasiment mais pas totalement symétrique. La légère asymétrie est probablement due à des différences de serrage de chaque côté de la plaque et à des effets thermiques résultants de la découpe au chalumeau de la plaque d'origine. Les déformations longitudinales dans la soudure sont très nettement en traction tandis que les déformations transversales correspondantes sont en compression et que les déformationssuivant l'épaisseur sont généralement faibles. La figure 7 présente la variation des déformations longitudinales au travers de l'épaisseur. Des gradients de tension jusqu'à $2000 \times 10^{-6}$ par $\mathrm{mm}$ sont visibles dans la soudure.

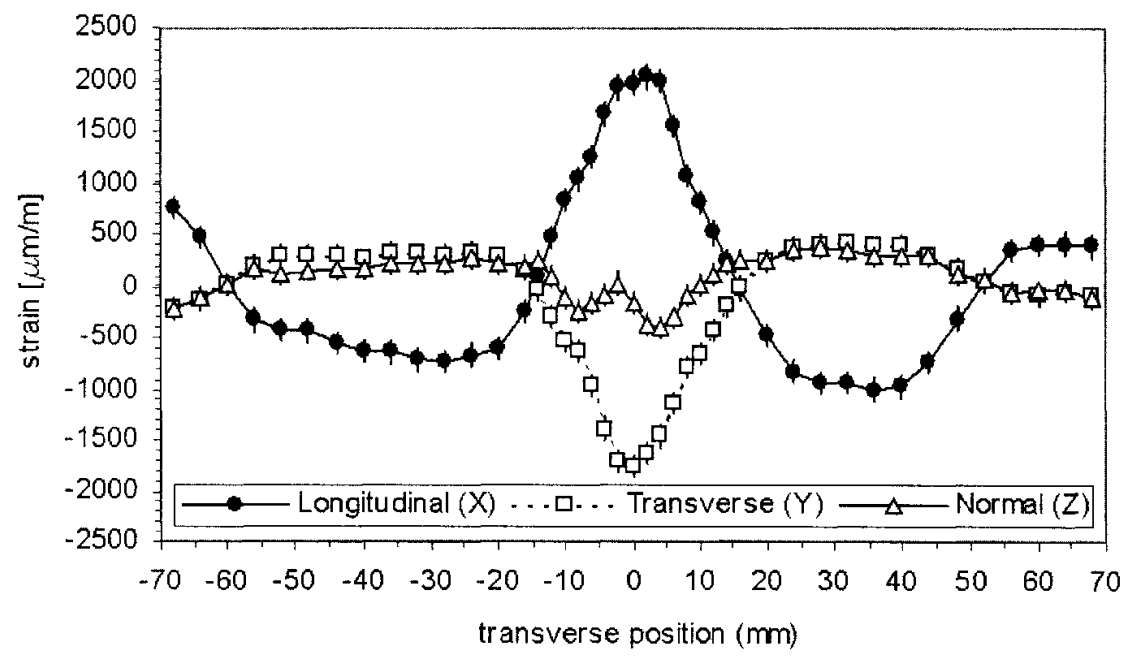

Figure 6 : Variation des déformations résiduelles le long de la ligne centrale de la soudure d'acier ferritique [15] 


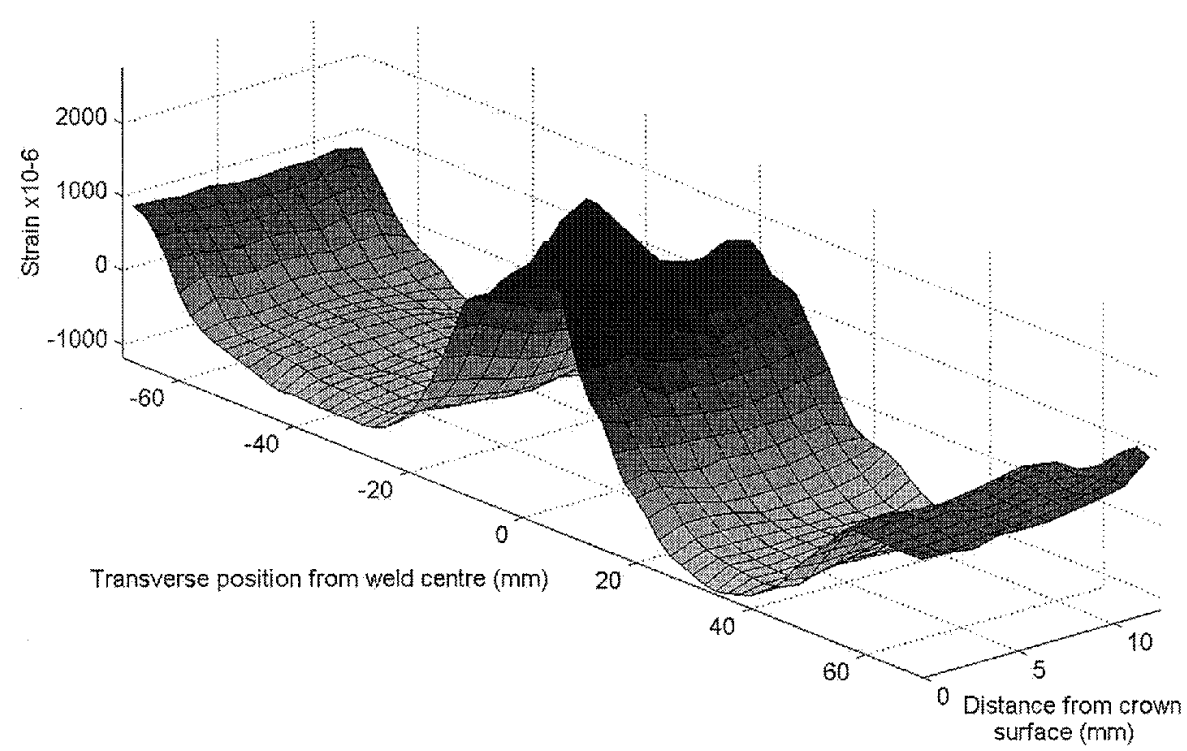

Figure 7 : Carte des zones de déformations résiduelles longitudinales suivant la coupe transversale YZ d'une soudure d'acier ferritique[16].

\subsection{Alliages d'aluminium soumis au grenaillage.}

\subsubsection{ALIAGE D'ALUMINIUM DE TYPE 7071 SOUMIS A UN FORT GRENAILLAGE}

Le grenaillage est bien connu pour générer un champ de contraintes résiduelles à gradient élevé qui est en compression en surface et contrebalancé par une tension au dessous de la surface. Les détails de ce champ de contraintes résiduelles dépendent du matériau de l'échantillon et des paramètres du grenaillage. Pour être bénéfique, la profondeur de la couche de compression doit être suffisante pour prévenir la propagation de fissures à partir de rayures et de défauts de surface. Il est difficile de mesurer de tels champs de contraintes résiduelles parce qu'ils sont généralement très limités et de courte portée $\sim 0.2 \mathrm{~mm}$. Il est possible de mesurer ces champs en utilisant des neutrons, mais pour des matériaux légers tels que l'aluminium les rayons $\mathrm{X}$ obtenus par synchrotrons ont leurs avantages.

Des mesures ont été réalisés à l'ESRF avec des rayons $\mathrm{X}$ de longueur d'onde $0.32 \AA$, les fentes délimitant les jauges ayant $0.1 \mathrm{~mm}$ de haut et de $5 \mathrm{~mm}$ de long. Ces mesures ont été faites sur un échantillon de plaque en alliage d'aluminium ayant subi un lourd grenaillage du type 7071. Pour la réflexion (113), la position $2 \theta$ du pic de diffraction était à $\sim 15^{\circ}$. Des mesures ont été faites suivant une orientation normale et jusqu'à une profondeur de $0.9 \mathrm{~mm}$ en dessous de la surface, là où l'atténuation du faisceau était de $\sim 90 \%$. Le profil du gradient élevé des déformations a été aisément résolu. En supposant que les déformations proches de la surface suivant une orientation normale sont inversement proportionnelles (par le coefficient de Poisson) aux déformations de surfaces dominantes, les déformations de surface 
peuvent être calculées à partir des déformations suivant la normale. La figure 8 illustre le champ des contraintes résiduelles estimé à partir de cette hypothèse en utilisant des rayons $X$ obtenus par synchrotron suivant la direction perpendiculaire [17]. Elle montre aussi pour comparer des données obtenues à partir de neutrons à résolution moyenne et la valeur de surface dérivée de mesures traditionnelles par rayons $X$ en quasi rétrodiffusion. L'accord entre les valeurs de surface données par les mesures faites à partir de rayons $X$ traditionnels et celles faites à partir de rayons $X$ obtenus à partir de synchrotrons est parfait. Mais la technique par neutrons a été incapable de résoudre tous les détails du profil des contraintes résiduelles à gradient variable.

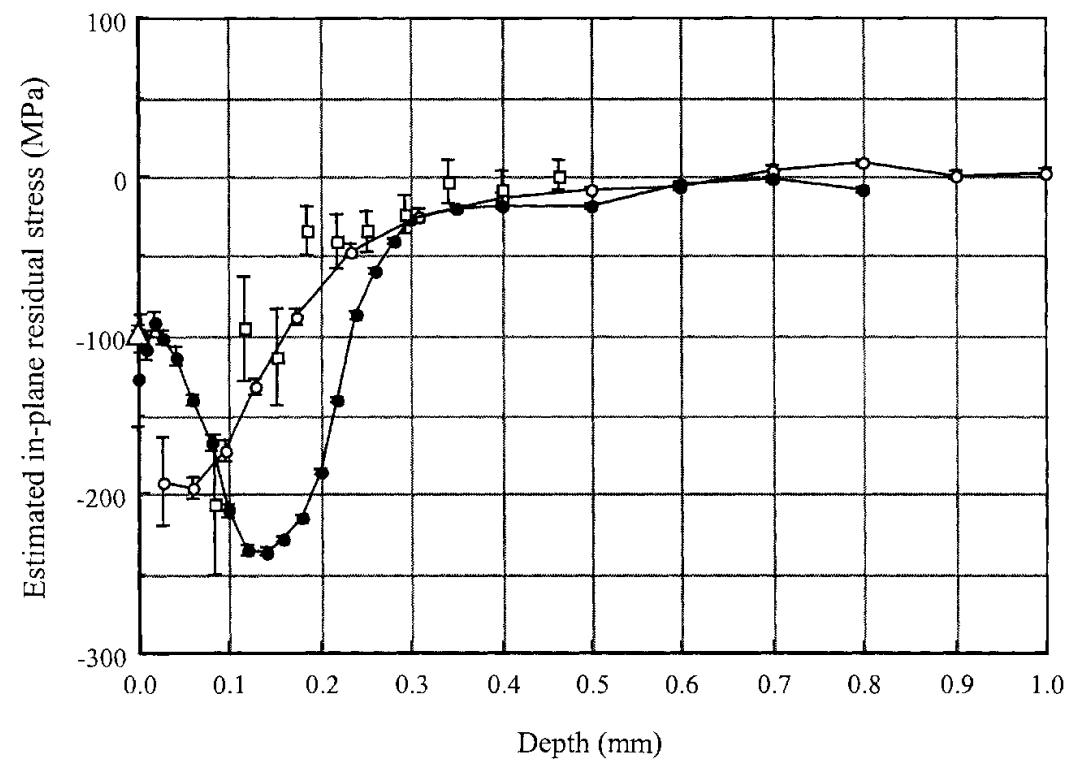

Figure 8:- Estimation des contraintes résiduelles de surface dans un alliage aluminium de type 7071 ayant été soumis à un lourd grenaillage - mesures par rayons Xobtenus par synchrotron, comparées à des mesures aux neutrons ou par rayons $X$ traditionnels [17].

\subsubsection{HIDUMINIUM SOUMIS A DIVERS GRENAILLAGE (DIFFERENCES D'INTENSITE)}

La figure 9 représente des champs de contraintes résiduelles de surface qui ont été mesurés au SRS de Daresbury au R.U. à partir de quatre échantillons de l'alliage aluminium dénommé Hiduminium [16]. Les résultats montrent clairement que la profondeur de la couche de compression augmente avec l'intensité du grenaillage.

\subsection{Trou agrandi a froid}

Le fuselage de la plupart des avions présente des milliers de rivets et de trous de rivets. Les trous sont des points de concentration des contraintes à partir desquels des fissures peuvent s'agrandir et se propager à l'usure. Systématiquement les trous de rivets dans un avion sont agrandis à froid en y faisant passer un mandrin dans un manchon, plus gros que la taille du 
trou évidemment. L'expansion génère au niveau du trou une relaxation en tensions qui, ellemême, génère un anneau de contraintes en compression qui résiste à la genèse et à la propagation de fissures.

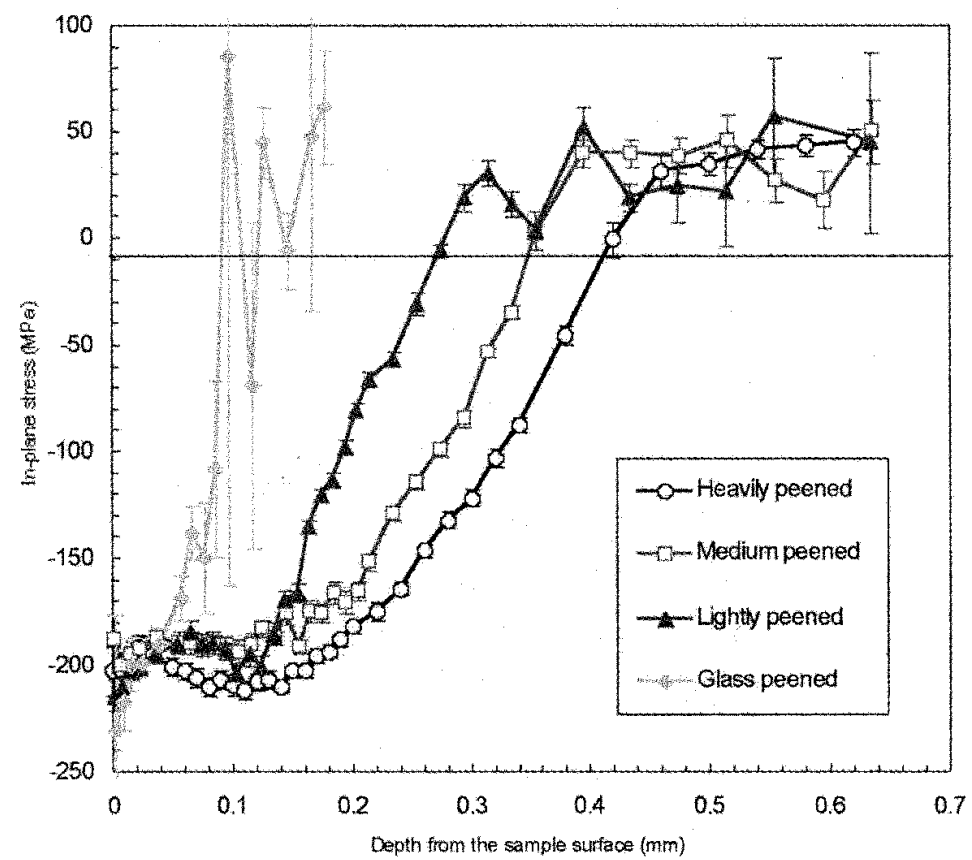

Figure 9 : Représentation graphique des contraintes résiduelles de surface dans l'Hiduminium soumis aux différents grenaillage selon l'intensité-mesures faites par rayons $X$ obtenus par synchrotrons.

Grâce aux équipements du NRC de Chalk River, on a fait des mesures aux neutrons de la variation radiale des déformations axiales, radiales et tangentielles autour d'un trou, agrandi de $4 \%$ à froid, dans une plaque d'alliage aluminium de type $7010 \mathrm{de} 11 \mathrm{~mm}$ d'épaisseur. Ces mesures ont été faites en utilisant la réflexion (113). Les résultats sont montrés par la figure 10. Les déformations tangentielles en compression du trou générées au perçage préviennent la propagation de fissures. 


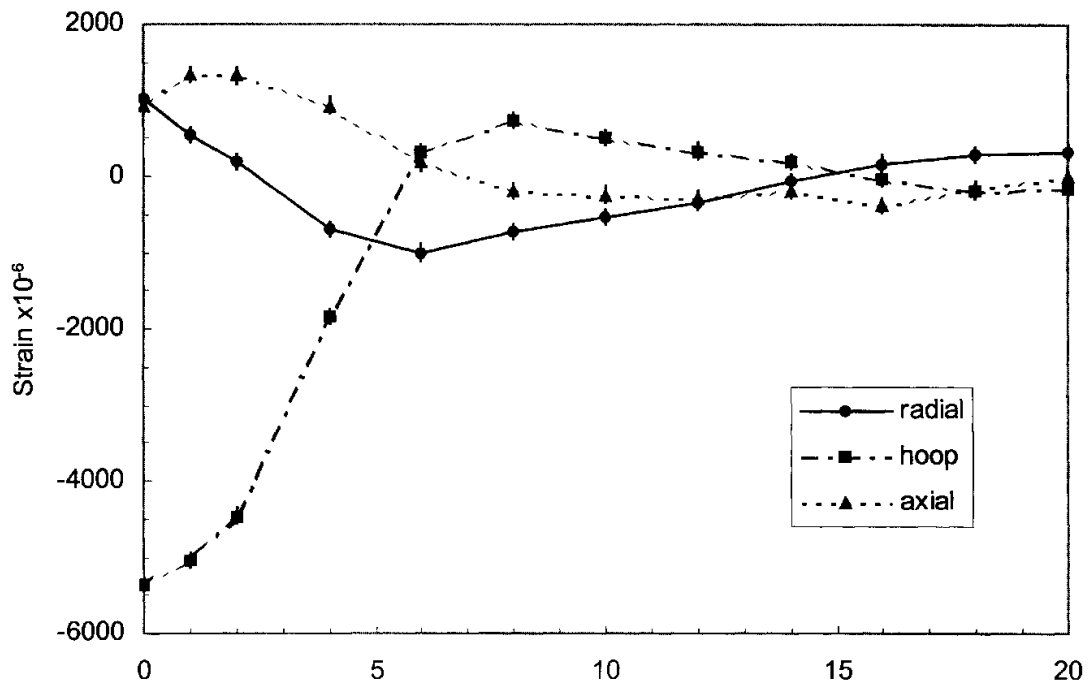

Figure 10:. Contraintes résiduelles autour d'un trou agrandi à froid [18]
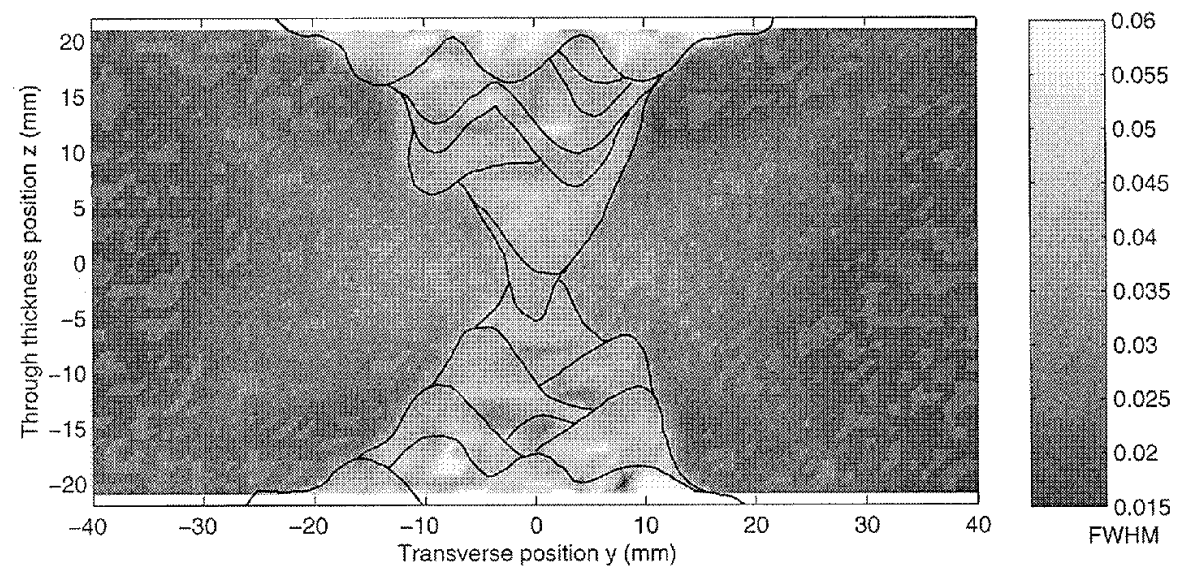

Figure 11. Carte dela largeur des pics de diffraction sur la coupe d'une soudure en alliage aluminium (les grains de la soudure sont visibles) [16] 


\subsection{Soudure en « double-V》- Microdéformations}

Les déplacements des pics de diffraction donnent une mesure de la variation de microdéformations dans un composant. La largeur des raies est une donnée de mesure des microdéformations, c'est-à-dire de la variation des déformations à l'échelle de microstructures. Les pics de diffraction de rayons $\mathrm{X}$ obtenus par synchrotron sont, par définition, très étroits et leur élargissement peut constituer un bon élément de mesure des microdéformations.

Des mesures ont été faites à l'ESRF sur la section transversale d'une soudure «multi-pass double-V » fournie par TWI. Des balayages ont été faits sur une matrice de $1.33 \mathrm{~mm}^{2}$ avec un faisceau de $1 \mathrm{~mm}^{2}$. Les résultats sont présentés en figure 11 . Il y a une corrélation évidente entre les grains de la soudure et la microtension : les déformations les plus élevées sont dans les grains les plus proches de la surface.

\section{Conclusion}

Les capacités non-destructrices et de pénétration de l'analyse des déformations par balayage par les neutrons sont désormais reconnues par les chercheurs en ingénierie, et la méthode commence à être plus largement acceptée par la communauté des ingénieurs et est reconnue comme outil de mesure. Le développement des premiers standards de mesure des contraintes résiduelles par neutrons incitera très certainement le monde de l'industrie à reprendre la méthode à son compte. On s'attend à ce que la méthode, plus nouvelle encore, des rayons $\mathrm{X}$ obtenus par synchrotron se développe rapidement comme une méthode complémentaire à haute résolution, surtout pour des mesures sur des matériaux avec des éléments légers. Les deux techniques sont particulièrement adaptées à la validation de champs de contraintes modélisés de façon numérique.

\section{Remerciements}

Le travail expérimental qu'on a présenté ici à été mis en place à l'ILL de Grenoble en France, et à l'AECL de Chalk River au Canada; les équipements ayant fourni les rayons $\mathrm{X}$ obtenus par synchrotron sont ceux du SRS de Daresbury au RU et de l'ESRF de Grenoble en France. L'aide financière à été fournie par l'EPSRC au RU. Nous remercions les membres des projets VAMAS TWA20 et RESTAND pour leurs contributions.

\section{References}

1. A Stacey, HJMacGillivray, GA Webster, $P J$ Webster, and $K R$ A Ziebeck: Measurement of residual stresses by neutron diffraction. J Strain Analysis 20 93-100 (1985)

2. PJWebster: The neutron strain scanner. Kerntechnik 56 178-182 (1990)

3. PJWebster: Spatial resolution and strain scanning. NATO Advanced Research Workshop on Measurement of Residual Stress using Neutron Diffraction, Oxford, March 1991. Measurement of Residual Stress using Neutron Diffraction (Kluwer Academic Publishers, The Netherlands, Eds. M T Hutchings and A D Krawitz ) 235-251 (1992) 
4. PJ Webster: The neutron strain scanner: Measurements in welds. IUTAM Symposium on Mechanical Effects of Welding, Luleå, 1991. Mechanical Effects of Welding (Springer-Verlag, Eds. L Karlsson, L-E Lindgren and M Jonsson) 143-150 (1992)

5. PJWebster, G Mills, $X$ Wang and W $P$ Kang: Residual stress measurements in rails by neutron diffraction. FRA/ERRI International Conference on Rail Quality and Maintenance for Modern Railway Operation, Delft, June 1992 (Rail Quality and Maintenance for Modern Railway Operation, Kluwer Academic Publishers, Eds. J J Kalker, D F Cannon, O Orringer) 307-314 (1993)

6. PJ Webster, G Mills, XD Wang, WP Kang, T M Holden and G F Modlen: Neutron diffraction study of residual stresses in extended components. Proceedings of the Fourth International Conference on Residual Stresses ICRS4, Baltimore, June 1994, 25-31 (1994)

7. PJWebster and $X D$ Wang: Neutron strain scanning of metal components. Surface Engineering 10 287-291 (1994)

8. $P J$ Webster, G Mills, XD Wang, WP Kang and T M Holden: Neutron strain scanning of a small welded austenitic stainless steel plate. J Strain Analysis 30 35-43 (1995)

9. PJWebster, GMills, XD Wang, WP Kang and TM Holden: Impediments to efficient through-surface strain scanning. J Neutron Research 3 223-240 (1996)

10. PJWebster: Strain scanning using $X$-rays and neutrons. Invited Presentation, European Powder Diffraction Conference, EPDIC IV, Chester, July 1995, (Proceedings of EPDIC IV - Part 1, Transtec Publications, Eds. R J Cernik, R Delhez and E J Mittemeijer) Materials Science Forum Vols. 228-231, 191-200 (1996)

11. PJ Webster, XD Wang and G Mills: Through-thickness strain scanning using synchrotron radiation. European Powder Diffraction Conference, EPDIC IV, Chester, July 1995, (Proceedings of EPDIC IV - Part 1, Transtec Publications, Eds. R J Cernik, R Delhez and E J Mittemeijer) Materials Science Forum Vols. 228-231, 227-232 (1996)

12. $P J$ Webster, G Mills and $X$ Wang: Residual strain scanning of engineering samples. SRS Annual Report 21/40 A65 (1992/93)

13. PJ Webster, G Mills and $X$ Wang: Residual strain scanning of engineering samples II SRS Annual Report 22/10 (1993/94)

14. P.J.Webster, G.Mills and X.Wang: Synchrotron strain scanning. SRS Annual Report 23/226 A598 (1994/95)

15. PJWebster: The status and future of residual stress analysis and the VAMAS TWA20 project. Proceedings of the ${ }^{\text {st }}$ International Symposium on Advanced Science Research ASR-2000, October 2000, Tokai, Japan (in press)

16. P $J$ Withers and $P J$ Webster: Neutron and synchrotron X-ray strain scanning. Strain $\mathbf{3 7}$ 19-33 (2001)

17. PJ Webster, G Mills, XD Wang and WP Kang: Synchrotron strain scanning through a peened aluminium alloy plate. Proceedings of ICRS5, July 1997, Linkoping, Sweden

18. PJWebster, G Mills, P A Browne, D J Hughes and TM Holden: Residual stresses around a cold-expanded hole. Proceedings of ICRS6, Oxford, July 2000, 125-132 (2000) 